\title{
Signaling Through Actin to Regulate Spine Formation and Function
}

\author{
Hirokazu Okada and Scott H. Soderling*
}

Department of Cell Biology, Duke University Medical School, Durham, North Carolina, 27710, USA

\begin{abstract}
Recent progress has greatly expanded our view of how signaling pathways regulate the actin cytoskeleton in post-synaptic spines. These studies reveal a complex interplay between pathways that highlight the role of the actin cytoskeleton during the development of spines as well as in response to stimuli that modify synaptic strength. This review discusses the results from these studies that include biochemical, cellular, and genetic approaches to understanding excitatory synapse formation and function.
\end{abstract}

Keywords: Actin, N-WASP, Spinogenesis, Synaptic Plasticity, WAVE-1, WRP, Dendritic Spine, Adhesion, Ephrin, Cadherin, AMPA, NMDA, LTP, LTD, Review.

\section{INTRODUCTION}

Dendritic spines are small protrusions that serve as postsynaptic specializations for the majority of excitatory synapses in the mammalian central nervous system $[1,2]$. The essential role of spines is believed to involve the spatial coordination of the biochemical events that occur in response to pre-synaptic inputs [3]. Mature spines typically have a round head and a narrow neck, and range in size from 0.5 to $2 \mu \mathrm{m}$ in length. While individual spines can vary widely in shape, the morphology of spines is generally classified into three categories: thin, stubby and mushroom. Recent studies indicate a strong correlation between spine morphology and synaptic strength [1, 3-5]. Additionally, a number of human mental retardation (MR) syndromes have been linked to altered spine morphology, including Fragile-X Syndrome [6, 7]. Thus, understanding the signals that instruct spine development and function is crucial to the understanding of synaptic mechanisms believed to underlie complex behaviors, including learning and memory.

The cytoskeletal structure that determines the morphology and function of spines is almost exclusively filamentous actin (F-actin) [8]. F-actin is a dynamic structure made up of double helical polymers formed from globular actin (Gactin) monomers. These monomers are arranged in a head to tail manner that gives actin filaments a polarity (one end called the barbed end and the other the pointed end). At steady state, addition of new G-actin monomers to F-actin filaments occurs predominantly at the barbed end of the filaments, and it is this addition that is thought to provide the force necessary to deform cellular membranes outward during changes in cell shape [9]. On the other hand, removal of G-actin from filaments in vivo occurs predominantly at the pointed end $[10,11]$.

The assembly of actin filaments in the cell is controlled by a variety of actin-binding proteins, some of which inhibit

*Address correspondence to this author at the Departments of Cell Biology, Duke University Medical School, Durham, N.C. 27710, USA;

E-mail: s.soderling@cellbio.duke.edu filament formation by monomer sequestration and others that encourage filament polymerization by nucleation. This balance represents an important regulatory step to modulate actin remodeling within the neuron. This review will discuss the signaling mechanisms believed to regulate actin dynamics during the development of spines (Fig. 1) and during mechanisms of synaptic plasticity (Fig. 2).

\section{ACTIN IS MODULATED BY RHO GTPASE SIGNAL- ING DURING SPINE DEVELOPMENT AND MATURATION}

Synaptic connections between appropriate neurons are critical for forming functional neural networks. Most pathways believed to regulate spine development appear to do so through Rho GTPase signaling (composed of 22 different GTPases, including Rho, Rac, and Cdc42) (Fig. 1). Rho GTPases function as molecular switches, cycling between the inactive GDP-bound and active GTP-bound states [12, 13]. Two families of proteins tightly control the temporal and spatial regulation of this switching; Guanine Nucleotide Exchange Factors (GEFs) and Rho GTPase Activating Proteins (GAPs). GEFs function as activators by increasing the GDP/GTP exchange rate of Rho-GTPases. GAPs stimulate the slow intrinsic GTPase activity of the Rho-GTPases and thus turn their signaling off.

The pivotal role of Rho GTPases in spine development was initially suggested by over-expression studies of mutant Rho GTPases. For example, constitutively-active Rac increases spine density in cultured neurons and in transgenic animals [14-16]. These spines often display an altered morphology, being wider and "veil-like" in appearance. In contrast, dominant-negative Rac leads to a reduction in spine density $[14,16]$. These spines are longer and have a filopodia-like appearance [17]. Expression of dominant-negative Rac in hippocampal slices also reduces the stability of spines that have already developed, suggesting a further role for Rac signaling in spine maintenance [17]. The effects of Rho largely oppose those of Rac. Constitutively-active Rho decreases spine density and length, while inhibition of Rho with $\mathrm{C} 3$ exoenzyme in hippocampal slices increase density and length $[14,16,18]$. Cdc42 is less well characterized [6] 


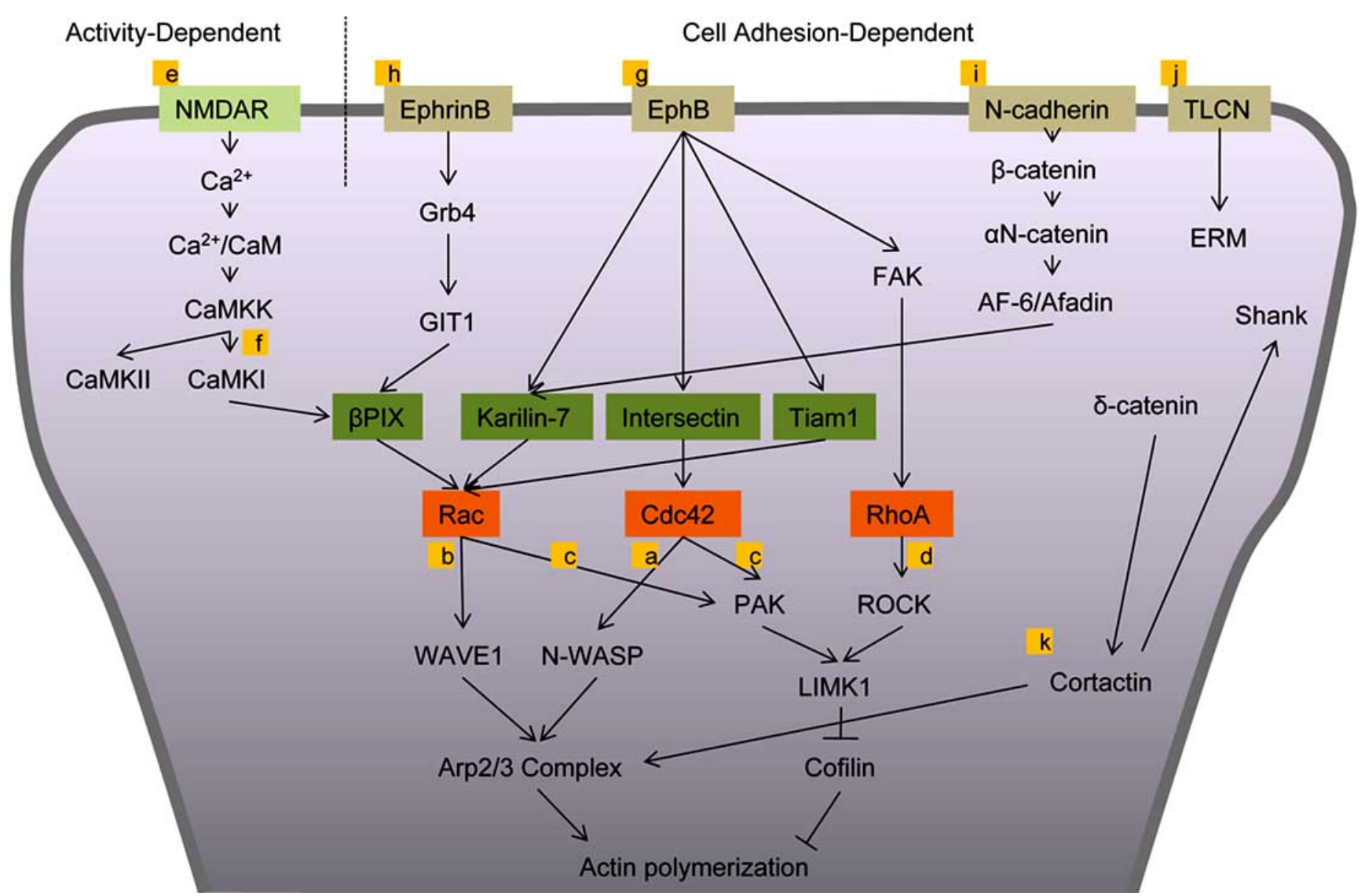

Fig. (1). Actin signaling in spine development and maturation. Activity-dependent signaling by calcium entry through NMDA receptors (NMDAR) (e) activates the Rac pathway via CaMKs and the Rac-GEF, $\beta$ PIX (f). Cell adhesion-dependent signaling originates from transsynaptic interactions of Ephrins (ligand) and Ephs (receptor) (g,h), or homophilic binding of N-cadherins (i) and Telencephalins (TLCN) (j). In Eph/Ephrin forward signaling (g), Rac, Cdc42 and RhoA pathways are activated (a - d) via three Rho GEFs (Kalirin-7, Intersectin and Tiam1) or by focal adhesion kinase (FAK). In reverse signaling (h), the Rac pathway is activated via $\beta$ PIX. N-cadherin activates Rac by Kalirin-7 (i). TLCN regulates actin through the ezrin/radixin/moesin (ERM) proteins (j). Cortactin regulates spine density in an Arp2/3 complex-dependent pathway (k).

but recent studies described below suggest the involvement of Cdc42 in signaling pathways that regulate spine morphology.

Rho GTPases regulate actin in dendritic spines through their downstream effectors [13]. Two members of the Wiskott-Aldrich syndrome protein (WASP) family, NWASP (neural WASP) and WAVE1 (WASP Verprolin homologous 1), function as molecular platforms for Arp2/3mediated actin nucleation downstream of $\mathrm{Cdc} 42$ and Rac signaling [19] (Fig. 1a and b). Both N-WASP and WAVE1 have a verprolin-cofilin-acidic region (VCA) domain that binds and activates the Arp2/3 complex [20]. N-WASP is auto-inhibited due to an intramolecular folding that masks the VCA domain [21]. Autoinhibition is relieved when activated $\mathrm{Cdc} 42$ binds N-WASP, allowing the N-WASP VCA domain to activate Arp2/3. Arp2/3 is a protein complex made of seven subunits, including the actin-related proteins (Arp2 and Arp3). This complex binds to the side of a preexisting actin filament and nucleates a new actin filament, forming a branch at an approximately 70-degree angle [22, 23]. Arp $2 / 3$ complex is enriched in dendritic spines, especially around the central core of the spines [24], and the involvement of signaling via Arp2/3 in spine morphology has been documented extensively. Reduction in the number of spines is observed with the knockdown of N-WASP, pharmacological inhibition of N-WASP, or the overexpression of a dominant-negative mutant of N-WASP $[25,26]$. WAVE1 purified from brain is inhibited by its tightly-associated proteins including CYFIP1, Nap1, Abi2, HSPC300, and WRP [27-29]. Activated Rac dissociates CYFIP1, Nap1 and Abi2 from the complex releasing active WAVE1 [27]. Loss of spine density has been observed in vivo or in isolated neurons from WAVE1 knockout mice [30, 31]. Inhibition of WAVE1 by Cdk5-mediated phosphorylation also decreases spine density [30]. Thus, the Arp $2 / 3$ complex can be activated downstream of either Rac or Cdc 42 through their distinct effectors and both pathways are critical for spinogenesis.

Rho GTPases also rapidly reorganize the spine actin cytoskeleton through kinase cascades. p21-activated kinases (PAK1-3), a family of serine/threonine protein kinases, phosphorylates LIM domain kinase 1 (LIMK1) downstream of Rac/Cdc42 signaling and thus couples Rho GTPases to actin cytoskeletal regulation through a LIMK1-cofilin pathway [32] (Fig. 1c). PAK is stimulated by binding to activated Rac1 or Cdc42, which destabilizes the autoinhibitory con- 


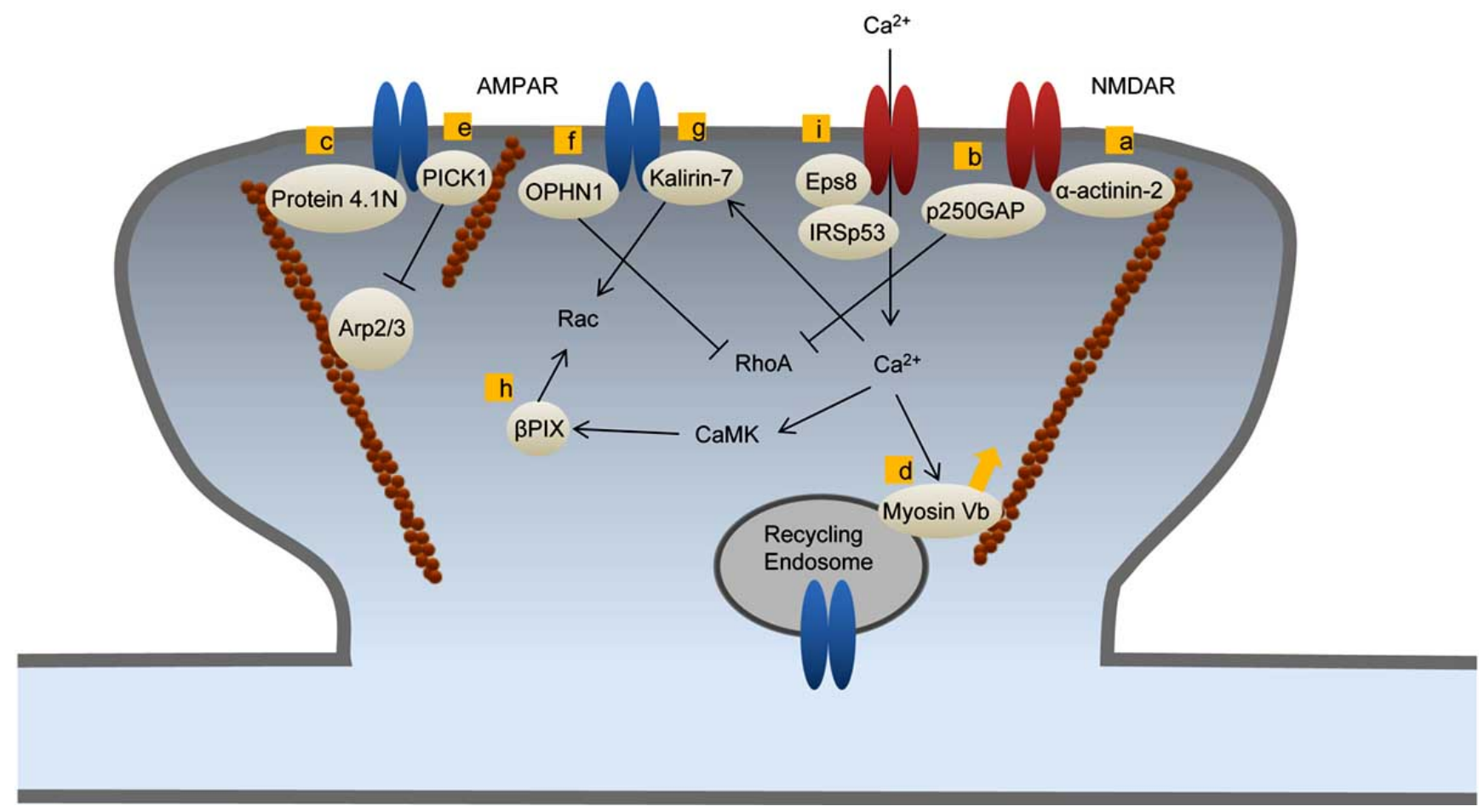

Fig. (2). Actin remodeling is linked to synaptic efficacy. $\alpha$-actinin-2 binds to NMDA receptors (NMDAR) and F-actin and regulates endocytosis of NMDAR (a). NMDAR also interacts with p250GAP (RhoA-GAP) and may influence actin cytoskeleton through RhoA signaling (b). Protein 4.1N and PICK1 bind to AMPA receptors (AMPAR) and F-actin and control AMPAR surface levels (c,e). PICK1 regulates AMPAR endocytosis by its ability to inhibit Arp2/3-mediated actin polymerization (e). Calcium entry through NMDAR activates myosin Vb and recruits recycling endosomes into spines, leading to enhanced exocytosis of AMPAR (d). Calcium influx also activates Rac signaling through Rac-GEFs (Kalirin-7 and $\beta$ PIX) and increases AMPAR trafficking at the postsynaptic surface (g,h). Oligophrenin-1 (OPHN1, RhoGAP) binds to AMPAR and stabilizes AMPAR surface levels, presumably by inhibiting RhoA signaling (f). Actin regulatory proteins, IRSp53 and Eps8, regulate NMDA receptor synaptic transmission (i).

formation of PAK homodimers [33]. Activated PAK1 and 3 are auto-phosphorylated at Thr423 and are highly enriched in spines [34]. Mice expressing a PAK autoinhibitory peptide in neurons of the forebrain show reduced spine density and larger spine heads [34]. Rho-associated (serine/threonine) protein kinase (ROCK) is also known to phosphorylate LIMK1 [35]. ROCK is primarily an effector of RhoA (Fig. 1d). Suppresion of ROCK activity with a specific chemical inhibitor (Y-27632) induces new, long spines with protrusive motility [17], which is consistent with the effect of RhoA inhibition.

Both PAK and ROCK activate LIMK1 by phosphorylation at Threonine 508 in the catalytic domain [32] and the actin filament-severing activity of cofilin is inhibited by phosphorylation of Serine 3 by LIMK1 [36, 37]. This reduces the affinity of cofilin for actin and as a result, inhibits cofilin's actin severing activity. The regulation of cofilin activity has been extensively implicated in the regulation of spine morphology. Cofilin binds ADP-bound actin monomers that are usually found in the older part of F-actin closer to the pointed end, and promotes the severing of filaments [38]. Cofilin is highly enriched at the tip of spine heads [39]. Furthermore, mice lacking LIMK1 display impaired spatial learning and elevated locomotor activity [40]. LIMK1 KO mice also show a reduced phosphorylation of cofilin in spines and have reduced spine head volumes and thicker spine necks [40], suggesting that LIMK1 stabilizes the actin filament network by inhibiting cofilin and allowing the formation of larger spine heads. Thus, the activity of cofilin may be negatively regulated through LIMK1 downstream of signaling by Rac, $\mathrm{Cdc} 42$, and RhoA.

\section{REGULATION OF RHO GTPASE SIGNALING DURING SPINE DEVELOPMENT AND MATURATION}

Relatively little is known about the signaling events that initiate the formation of dendritic spines during postnatal development. It is believed that excitatory synaptogenesis starts from the transient formation of thin, long filopodia-like membrane protrusions on the dendritic shafts [2, 41, 42]. These protrusions can last for minutes to hours. One recent study suggests an important role for Rif, a member of Rho GTPase family, in the elongation of dendritic filopodia [43]. Using an assay to visualize barbed end actin polymerization in cultured hippocampal neurons, it was shown that filopodia-like spine precursors elongate through actin polymerization at both the tip and dendritic root of the filopodia-like structure. Thus, actin in developing spines appears to be organized in an anti-parallel manner. mDia2, a member of the formin family of actin nucleators, is enriched at the tip of these protrusions and regulates actin polymerization downstream of Rif. This fits well with the known ability of formins to enhance the polymerization of straight actin filaments, similar to those that are bundled within filopodia [44]. Whether formins instruct actin dynamics at later time points in mature spines is not yet clear, but seems likely. 
Synapse formation is initiated when protrusions make a contact with a presynaptic terminus via cell-cell adhesions or chemically via released neurotransmitters. Once productive contact has been made, these protrusions are transformed morphologically into mature spines (with a terminal head that is at least twice the width of the spine neck). In mammals, spine density in vivo increases in early life and reaches a maximum when synaptic plasticity is at its peak. Subsequently it decreases slightly and then remains relatively constant throughout later life [41]. The temporal profile of spine development and pruning may vary by brain region and species. In rat hippocampus, spine number reaches a peak during the third postnatal week and then stays relatively constant [2]. Spine formation and retraction can continue to occur in the adult brain and it may be that the turnover rate depends on the brain region and age. It is estimated that in adult mice (4-6 month old), $3-5 \%$ of spines experience retraction and formation over 2 weeks in various cortical regions [45].

The formation of new filopodia-like protrusions on dendritic shafts can also be tuned by patterns of synaptic activity [46]. Time-lapse two-photon imaging has shown that LongTerm Potentiation (LTP)-inducing high-frequency synaptic stimulation to CA1 hippocampal pyramidal neurons induces the rapid formation of filopodia-like protrusions in an $\mathrm{N}$ methyl D-aspartic acid receptor (NMDAR)-dependent manner [47] (Fig. 1e). One kinase that is activated downstream of NMDAR is $\mathrm{Ca}^{2+} / \mathrm{CaM}$-dependent protein kinase II (CaMKII) [48]. Intracellular application of active CaMKII induces rapid filopodia-like growth, while inhibition of endogenous CaMKII activity prevents filopodia-like protrusions from forming [49]. Thus CaMKII signaling, potentially during LTP, may play an essential role in activity-dependent stimulation of new spine growth.

Once dendritic filopodia-like protrusions make contact with pre-synaptic terminals, only some advance to form spines. Time-lapse calcium imaging reveals that local dendritic calcium transients occur within seconds after contact and these contacts are stabilized only when the frequency of the transients is high [50]. This indicates a significant role of calcium entry in triggering the transformation of dendritic filopodia-like protrusions into mature spines. Downstream of calcium entry, $\mathrm{Ca}^{2+} / \mathrm{CaM}$-dependent protein kinase kinase (CaMKK) and CaMKI form a protein complex with $\mathrm{G}$ protein-coupled receptor kinase-interacting protein (GIT1) and the Rac-GEF, $\beta$ PIX (PAK-interacting exchange factor beta) [51]. Calcium entry stimulates CaMKK and CaMKI, and CaMKI in turn activates $\beta$ PIX via phosphorylation at Ser516, leading to Racl activation (Fig. 1f). Suppression of CaMKs or expression of an inactive $\beta$ PIX mutant in cultured hippocampal neurons or in organotypic hippocampal slices reduces spine formation. Constitutive-active PAK1, which is downstream of $\beta$ PIX and Rac, rescues the spine reduction [51]. Thus, a CaMK-GIT1- $\beta$ PIX complex that activates the Rac1-PAK pathway is also involved in spine formation following activity-dependent calcium entry.

Signaling by cell adhesion molecules following contact between dendritic filopodia and axons also regulates the transformation of filopodia-like protrusions into bulbous spines. Bi-directional signaling (forward and reverse signaling) through trans-synaptic Eph (receptor)/Ephrin (ligand) interactions is one of the best characterized molecular mechanisms for this event [52]. In forward signaling, presynaptic Ephrins interact with postsynaptic Eph family receptor tyrosine kinases (Ephs) and induce signaling downstream of Ephs (Fig. 1g). Expression of dominant-negative forms of EphBs that are kinase-inactive or deficient for the cytoplasmic domain in dissociated hippocampal neurons increases filopodia-like, headless protrusions at the expense of mature spines. On the contrary, activation of forward signaling by the application of preclustered EphrinB ectodomains fused to the Fc region of human IgG (EphrinB-Fc) induces dendritic spine maturation $[25,53,54]$. Combining multiple EphB KO mice reveals the functional redundancy of EphBs in spine morphogenesis [53]. Triple EphB (EphB13 )-deficient mice show a significant reduction of spine density and impaired spine morphogenesis leading to headless or small-headed spines. Cultured hippocampal neurons from the triple $\mathrm{KO}$ mice exhibit long, thin dendritic filopodia and a total loss of mature spines. The re-expression of EphB2 is sufficient to rescue the abnormal spine morphology in the triple KO cultured neurons [53].

Four distinct pathways have been reported to function downstream of EphB to regulate spine morphogenesis (Fig. 1g). Activation of EphBs causes phosphorylation of three Rho GEFs (intersectin, kalirin-7 and Tiam1), leading to Rho GTPase-mediated actin cytoskeletal remodeling. Intersectin, phosphorylated by EphB2, activates Cdc42 and induces actin polymerization through the N-WASP-Arp2/3 complex pathway [25]. EphB2 activation by clustered EphrinB1 (EphrinB1-Fc) recruits Kalirin-7, a Rac-GEF, to synapses and activates the Rac1-PAK (and presumably the subsequent LIMK1-cofilin) pathway [54]. Activated EphBs recruit Tiam1, another Rac-GEF, to an EphB complex containing NMDA receptors and phosphorylate Tiam1 (and presumably activate a Rac-dependent pathway) [55]. Recent work has shown that focal adhesion kinase (FAK), a nonreceptor tyrosine kinase, acts downstream of EphB2 and regulates cofilin activity through the RhoA-ROCK-LIMK1 pathway [56]. Thus, these signals seem to converge on the enhancement of actin polymerization, presumably through the activation of Arp2/3 complex or the inhibition of cofilin. Yet the spatiotemporal regulation of these pathways in vivo remains to be determined.

In reverse Ephrin signaling, the binding of Ephs to Ephrins activates signaling in cells expressing Ephrins (Fig. 1h). At some synapses such as those of the hippocampal CA3CA1 region, EphrinBs have been observed in dendrites and spines [57, 58]. A recent report has shown that clustered EphB2 (EphB2-Fc) application to cultured hippocampal neurons reduces filopodia-like protrusions and increases mature spines with mushroom-like heads [59]. Interference with EphrinB1 signaling by expressing dominant-negative EphrinB1 that lacks the cytoplasmic domain, causes a marked increase in headless filopodia-like protrusions, indicating an indispensible role of reverse signaling for proper spine development. Grb4 (SH2 and SH3 domain-containing adaptor protein) and GIT1 appear to function downstream in this reverse pathway. Upon EphrinB activation, GIT1 is phosphorylated at Tyr392. Grb4 then binds to the phosphorylated GIT1 by its SH2 domain and recruits GIT1 to synapses [59]. Considering that GIT1 forms a signaling complex with $\beta$ PIX [51], $\beta$ PIX might act downstream of the GIT1/Grb4 com- 
plex. Time-lapse imaging also reveals another role for EphB signaling in dendritic filopodial motility [60]. This motility is necessary for dendritic filopodia to search for and make connections to presynaptic partners. Thus EphB signaling regulates multiple aspects of spine formation from filopodia motility to spine maturation.

Cadherins mediate cell-cell adhesion through calciumdependent homophilic binding [61] (Fig. 1i). N-cadherin is a neuronal cadherin that, in nascent synapses, is clustered on both pre- and post- synaptic membranes, but outside of the postsynaptic density (PSD) in mature synapses [62]. Catenins bind to the cytoplasmic tail of $\mathrm{N}$-cadherin and may mediate its interaction with the actin cytoskeleton. $\beta$-catenin directly binds to cadherin and bridges the interaction between cadherin and $\alpha \mathrm{N}$-catenin. $\alpha \mathrm{N}$-catenin can either directly interact with actin, or may be linked to actin by binding other proteins such as $\alpha$-actinin or spectrin [63]. Loss of $\alpha \mathrm{N}$ catenin in hippocampal neurons increases the formation of filopodia-like protrusions with enhanced mobility. In contrast, overexpression of $\alpha \mathrm{N}$-catenin increases spine density and biases the morphology towards enlarged spine heads [64]. Acute deletion of $\beta$-catenin in cultured hippocampal neurons alters spine morphology such that they are thin and elongated [65]. $\delta$-catenin is exclusively expressed in neurons and is also implicated in spine morphogenesis. Knockdown of $\delta$-catenin in cultured hippocampal neurons increases spine density at early stages (Days in vitro (DIV) 11-17) but decreases at later stage (DIV 21) [66, 67]. Interestingly $\delta$-catenin binds to cortactin and the association between $\delta$-catenin and cortactin may be regulated by metabotropic glutamate receptor (mGluR) signaling $[66,67]$. This may be important because cortactin also interacts with shank and connects F-actin to the PSD [68]. Immunoelectron microscopy indicates co-localization of cortactin with Arp2/3 complex in the central core of hippocampal spines and cortactin is known to stabilize Arp2/3 dependent actin filaments [24]. RNAi knockdown of cortactin in hippocampal neurons also leads to reduced spine density [69] (Fig. 1k).

Recently, a link between N-cadherin and Rho GTPase signaling has also been revealed [70] (Fig. 1i). Clustered Ncadherins recruit kalirin-7 to synapses through the scaffolding protein AF-6/afadin. AF-6/afadin is localized to cadherin adhesion junctions by its interactions with $\alpha$-catenin and nectin. Activation of $\mathrm{N}$-cadherin by preclustered $\mathrm{N}$-cadherin (Fc-N-cad) increases Racl in spines and PAK phosphorylation, following the Kalirin-7 recruitment. This results in spine enlargement in cultured cortical pyramidal neurons. Disruption of $\mathrm{N}$-cadherin adhesion using antibody against the extracellular $\mathrm{N}$-cadherin domain reduces Rac1 in spines and leads to long, thin spines [70]. These results indicate a significant role of cadherin-catenin systems in spine formation.

Telencephalin (TLCN) has recently been implicated in spine formation. TLCN is an immunoglobulin (Ig) superfamily cell adhesion molecule specifically expressed in the telencephalon in mammalian brains, which is the rostral brain region including cerebral cortex and hippocampus [71] (Fig. 1j). In neurons, TLCN is highly enriched in dendritic filopodia-like protrusions but excluded from mature spines [72]. Deletion of TLCN in vivo or in cultured hippocampal neurons causes enhanced spine maturation and reduced den- dritic filopodia-like protrusions. Overexpression of TLCN results in the formation of nascent filopodia-like protrusions and the reversion of mature spines to these structures [72]. The cytoplasmic domain of TLCN interacts with ERM (ezrin/radixin/moesin) family of actin-binding proteins and $\alpha$-actinin [73]. Knockdown or overexpression of constitutive-active ERM proteins also modulates the formation of dendritic filopodia. Several kinases including Rho-dependent kinases are known to phosphorylate ERM proteins, but it remains unclear which kinases function downstream of TLCN. The role of TLCN is unique compared to other celladhesion molecules since TLCN negatively regulates the maturation from dendritic filopodia to bulbous spines.

In summary, there is a complex interplay between activity-dependent and adhesion mediated signaling that mediates the development and maturation of spines (Fig. 1). These pathways ultimately converge on the actin cytoskeleton by regulating the activity of Rho GTPases by altering the localization and activity of GEFs. Actin is not just limited to regulating the development and formation of excitatory synapses. It plays a key instructive role in translating patterns of activity into long-lasting changes in synaptic efficacy.

\section{ACTIN REMODELING IN THE SPINE IS INTIMATELY PAIRED WITH SYNAPTIC ACTIVITY AND STRUCTURAL PLASTICITY}

In 1973 Long-Term Potentiation (LTP) of synaptic transmission was described in the dentate gyrus after the high-frequency stimulation of the perforant path [74]. Shortly thereafter, it was observed by electron microscopy (EM) that tetanic stimulation induced long lasting enlargement of dendritic spines [75]. These structural changes in spine morphology were postulated to form the basis for longterm information storage inherit to the process of memory formation. Yet how spines altered their morphology was unknown. In 1982 Francis Crick speculated the answer to this question could have important implications for the mechanisms of learning and memory and he posed the question "Suppose actin was discovered in spines?" [76]. That year two groups showed actin, but not tubulin was highly enriched in the spine, supporting the theory that actin remodeling would be linked to mechanisms of stable changes associated with enhanced synaptic transmission [77, 78]. LongTerm Depression (LTD), induced by low frequency stimulation in the hippocampus was described later, demonstrating that long lasting changes in synaptic efficacy can be modified bi-directionally [79]. These two characteristic long-term changes at the postsynaptic spine are: 1) structural plasticity - the enlargement/shrinkage in spine shape or the creation/loss of spines; and 2) LTP/LTD -increased or decreased efficacy of neurotransmission. These are believed to represent the mechanisms of information storage in the CNS.

Actin remodeling is required for both structural plasticity and LTP/LTD. Inhibiting actin polymerization blocks the stable expression of LTP as well as spine morphological changes, suggesting actin remodeling is a central event to the formation of stable modifications in synaptic efficacy [8083]. Many recent studies have illuminated how the actin cytoskeleton is spatially and temporally organized within the spine. Ultrastructural studies show actin filaments within the spine are organized such that some emerge from below the 
PSD as well as being present throughout the spine head [84]. Labeling the actin filaments with the myosin S-1 fragment shows actin forms a beautiful lattice throughout the spine head with the barbed ends oriented towards the PSD and plasma membrane [77]. Studies of fluorescent actin using Fluorescent Recovery After Photobleaching (FRAP) indicate actin is highly dynamic within the spine, with roughly 85 percent of actin turning over within two minutes [85]. Because actin rapidly turns over and it is the primary cytoskeleton component of the spine, it is well-suited to mediate the cytoarchitectural and functional changes that occur in the spine in response to synaptic activity. Indeed, studies are now revealing how the regulation of actin is intimately paired with changes in long-lasting synaptic strength. Visualization of F-actin using fluorescent phalloidin in tissue sections indicates that theta-burst stimuli that induce LongTerm Potentiation lead to increases in spine actin polymerization [86]. This increase in F-actin content is stable for long periods of time and like LTP is NMDA receptor dependent. Actin appears to play a critical role in the consolidation of LTP during the first 30 minutes after induction, and this consolidation may depend on integrin-dependent adhesion and signaling [87]. Similar increases in spine actin polymerization are observed for up to five weeks using in vivo LTP induction protocols in freely mobile mice, suggesting activity coupled increases in spine F-actin are physiologically relevant and long-lasting [80]. FRET analysis using YFP and CFP labeled actin to quantify the ratios of F-actin and Gactin show that tetanic stimulation to generate LTP induces spine actin polymerization [88]. Conversely, low-frequency stimulation associated with LTD induction shifts the ratio towards G-actin or actin depolymerization and is associated with spine head shrinkage. Thus actin dynamics appear to be coordinated bi-directionally with synaptic morphology and transmission efficacy (Fig. 2).

Although the spine is a relatively small structure, it appears to be exquisitely organized at the functional level. Emerging studies suggest spatially distinct pools of actin accomplish dynamic remodeling of the spine cytoskeleton. Under basal conditions there are two pools of actin: a dynamic fraction of actin that is localized along the tip of the spine; and a stable pool of actin that is concentrated at the center and base of the spine [85, 89]. Upon activation by glutamate, a new pool of actin is induced. This third pool of actin is referred to as the enlargement pool as it is required for the physical enlargement of the spine that is associated with LTP. The enlargement pool of actin fills the spine head and requires CaMKII for its stable expression. Interestingly, some spines do not show a stable increase in volume after potentiation. Remarkably these spines appeared to consistently "dump" actin through the spine neck into the dendritic shaft. It was speculated that the diameter of the spine neck, might be an important determinate as to whether stable longterm structural plasticity was maintained. Interestingly this possibility harkens back to early speculations that changes in the thickness of the spine neck (then termed "stem") could provide a means to information storage by altering the confining properties of the spine head [90]. The spatial arrangement within the spine of different actin pools potentially correlates well with the sub-spine distribution of actin remodeling proteins as assessed by immuno-EM [24, 39, 91]. These ultrastructural studies show that cofilin, which is re- quired for actin remodeling, is enriched at the tip of the spine. Cortactin, which stabilizes actin is enriched at the center. Interestingly, Arp $2 / 3$ is enriched as a hollow ring around the middle cortex of the spine. Thus one could speculate that cofilin may be important for regulating the dynamic pool, cortactin the stable pool, and Arp $2 / 3$ mediated actin remodeling may be critical for the enlargement pool. This also fits with the well-characterized ability of Arp $2 / 3$ to rapidly induce new actin filament polymerization.

Finally, the synaptic localization of actin regulatory proteins may also be directly regulated by synaptic activity. The best example of this activity dependent redistribution is for the G-actin binding protein profilin. Profilin labeled with GFP is robustly recruited to dendritic spines in cultures treated with glutamate, and inhibitors of NMDA, but not AMPA or metabotropic glutamate receptors, block this spine enrichment [92, 93]. Interestingly, GFP-profilin is recruited to spines in response to both theta-burst and low frequency stimulation, suggesting elevated profilin within the spine is equally important for LTP and LTD. The localization of profilin in response to fear conditioning, a learning and memory behavior that involves the amygdala, has also been examined in vivo by immuno-EM [94]. In these experiments, fear conditioning was associated with an increase in profilin in spines within the lateral amygdala when compared to naïve or unpaired stimulus trained animals. Recently it has also been shown that Oligophrenin-1 (OPHN1), which is a RhoGAP implicated in mental retardation, is enriched in spines during LTP $[95,96]$. This synaptic targeting of OPHN1 is mediated by an $\mathrm{N}$-terminal $\mathrm{PH}$ domain and is important for regulating AMPAR trafficking (see below).

\section{HOW IS ACTIN REMODELING LINKED TO ALTERED SYNAPTIC EFFICACY?}

It is generally accepted that synaptic cytoskeletal changes are intimately paired with mechanisms believed to underlie information storage. Yet how may actin facilitate altered synaptic strength at the mechanistic level? LTP and LTD, the molecular correlates of learning and memory, are manifest by enhanced or depressed field excitatory post-synaptic currents (fEPSCs) after high or low frequency stimulation respectively. Because the majority of fEPSCs evoked by LTP and LTD are mediated by AMPA type glutamate receptors (AMPARs), trafficking of AMPAR subunits at the synaptic membrane is one key mechanism for regulating both LTP and LTD [97-99]. For example, a dynamic pool of extrasynaptic AMPA receptors is available for rapid delivery into the synapse upon induction of LTP [100]. Conversely, endocytic removal of perisynaptic AMPA receptors precedes the loss of synaptic AMPA receptors that occur upon LTD induction [101]. Additionally, rapid loss of NMDAR during LTD has also been observed in vitro and in vivo [102-104]. Thus, altering the surface levels of glutamate receptors is believed to be one of the central events that modify experience dependent synaptic connectivity during learning and memory [100, 105-107].

Both NMDA and AMPA type glutamate receptors organize signaling complexes of Rho-GTPase regulatory proteins and are indirectly linked to actin by binding several cytoskeletal proteins. For example, the actin binding and crosslinking protein, $\alpha$-actinin-2, binds to NMDA receptor 
subunits NR1 and NR2B [108] (Fig. 2a). $\alpha$-actinin-2 is concentrated throughout the PSD and binds NR1 within a membrane proximal cytoplasmic tail that is required for endoystosis $[109,110]$. Use dependent rundown of the NMDAR is also blocked by stabilizing the actin cytoskeleton or enhanced by inducing actin depolymerization [111]. These data suggest tethering the NMDAR to the actin cytoskeleton regulates aspects of its surface turnover. Consistent with this possibility, RhoA activity regulates NMDA rundown via regulation of the actin cytoskeleton and NR2B interacts with the RhoA GAP p250GAP [112-114] (Fig. 2b). How p250GAP is regulated in the context of NMDA signaling is not yet clear, but recent work shows it is regulated at the translational level by the microRNA 132 in an activity dependent manner [115].

AMPA receptors also organize Rho-GTPase signaling and are tethered to the actin cytoskeleton. The GluR1 AMPA receptor associates with the neuronal membrane-actin adaptor protein, 4.1N [116] (Fig. 2c). This interaction occurs between the membrane proximal region of GluR1 and a CTerminal Domain (CTD) of 4.1N. Overexpression of the CTD, which binds GluR1 but does not interact with the actin cytoskeleton, reduces GluR1 surface levels. Disruption of the actin cytoskeleton also reduces AMPAR surface levels $[82,117]$, suggesting the coupling of the GluR1 to actin via $4.1 \mathrm{~N}$ regulates its trafficking. The ability of $4.1 \mathrm{~N}$ to modulate synaptic levels of GluR1 is highly regulated since the interaction between GluR1 and $4.1 \mathrm{~N}$ is regulated by palmitoylation and PKC phosphorylation of GluR1 [118]. Recent work has extended these observations using live Total Internal Reflection Fluorescence (TIRF) imaging of surface GluR1 using Super-Ecliptic pHluorin GFP (SEP-GFP) GluR1 [118]. SEP-GFP is sensitive to $\mathrm{pH}$ and only emits fluorescence when exposed to the neutral extracellular imaging medium. This in combination with TIRF allows for direct imaging of the addition or removal of receptors at the membrane. These studies show the $4.1 \mathrm{~N}$ interaction is required for activity driven insertion of GluR1 into extrasynaptic sites. Importantly, knockdown of $4.1 \mathrm{~N}$ impairs LTP maintenance.

How does actin facilitate the insertion of AMPAR into postsynaptic sites during LTP? Previous work showed that recycling endosomes are recruited to spines during LTP induction and that recycling endosome-dependent AMPAR trafficking is required for LTP [119]. Activity dependent spine trafficking of organelles is likely to depend on both actin-based motors and calcium signaling. Recent work shows Myosin $\mathrm{Vb}$, an actin-based motor that is activated by elevated calcium, mediates the recruitment of recycling endosomes to dendritic spines and is required for GluR1 insertion into postsynaptic sites during LTP [120] (Fig. 2d). This actin-based transport is required for LTP in vivo, since transgenic mice expressing a form of Myosin $\mathrm{Vb}$ that can be acutely inhibited also show a complete loss of LTP in the presence of inhibitor, but not vehicle. Interestingly, structural plasticity of spines also depended on Myosin $\mathrm{Vb}$, suggesting the addition of membrane from recycling endosomes is required for both structural plasticity and LTP. Thus, the actin cytoskeleton organizes a network of filaments critical for dynamic recruitment of recycling endosomes via Myosin Vb. This specifically enhances exocytosis of AMPAR containing membrane to synaptic sites.
Different AMPA type subunits also organize regulators of the actin cytoskeleton that directly influence their trafficking, including endocytosis. PICK1, a BAR and PDZ domain containing protein interacts with the AMPA receptors GluR2/3 and this interaction is required for AMPAR endocytosis in response to NMDAR activation [121-123] (Fig. 2e). PICK1 also contains an actin binding site within the BAR domain and a newly identified acidic region that interacts with the Arp2/3 complex [124]. As noted above, Arp2/3 is believed to be a major regulator of de novo actin polymerization in the spine. PICK1 binding to Arp2/3 inhibits its activation by N-WASP, suggesting PICK1 binds competitively without activating Arp2/3. Binding of PICK1 to Arp2/3 requires Tryptophan 413 within the acidic domain, which is analogous to a conserved tryptophan found within the Arp2/3 binding region of N-WASP, WAVE1, and cortactin. Mutation of this tryptophan abolishes the ability of PICK1 to bind to and inhibit Arp2/3. Importantly, expression of this mutant form of PICK1 blocks NMDA-induced AMPAR endocytosis; Latrunculin A, a toxin that inhibits actin polymerization, reverses this effect. Together these results strongly argue the inhibition of Arp2/3-mediated actin polymerization is required for AMPAR internalization. This is somewhat surprising, as in non-neuronal cells it has been shown that actin and Arp $2 / 3$ are transiently recruited to clathrin-mediated endocytosis events just before vesicle scission from the plasma membrane $[125,126]$. Inhibition of activators of Arp2/3, such as N-WASP, impairs endocytosis in non-neuronal cells [127]. Thus either stimulated AMPAR endocytosis has different requirements for actin polymerization in the spine compared to non-neuronal cells, or more likely there are spatial-temporal requirements for both activation and inhibition of Arp2/3 during internalization. More work will be required to distinguish these possibilities.

AMPAR trafficking is also regulated by Rho-dependent signaling via the Rho-GAP OPHN1 [96] (Fig. 2f). OPHN1 is part of a GluR1/2 complex and knockdown of OPHN1 leads to a significant reduction in LTP. These effects are likely to be due to altered AMPAR trafficking since overexpression of OPHN1 stabilizes surface levels of GluR1/2 and blocking GluR1/2 internalization occludes the effect of OPHN1 knockdown on AMPAR mediated synaptic transmission. The regulation of AMPAR by OPHN1 is dependent on the Rho-GAP domain of OPHN1, and inhibition of Rho-Kinase affected surface GluR $1 / 2$ stability similar to the overexpression of OPHN1. Because OPHN1 can be recruited to spines during LTP, it may form a positive feedback loop by inhibiting Rho - Rock signaling to functionally stabilize surface AMPAR levels. The ability of OPHN1 to influence glutamate receptor trafficking may be one important clue as to the mechanisms by which loss of OPHN1 leads to mental retardation in humans [128]. Interestingly, Rac signaling also regulates LTP via the actin cytoskeleton and AMPAR trafficking. Induction of LTP causes an NMDA-dependent activation of Rac via the GEFs Kalirin-7 and $\beta$ PIX (Fig. 2g and h). Activation of these GEFs is mediated by NMDA dependent activation of CaM Kinases [51, 129]. Like OPHN1, Kalirin-7 also associates with GluR1 and knockdown of Kalirin-7 reduces the levels of GluR1 in the spine and AMPARmediated basal synaptic transmission [129]. Knockdown of Kalirin-7 also impairs NMDA-dependent delivery of GluR1. Because both a Rho-GAP and Rac-GEF appear to associate 
with and regulate AMPAR trafficking, how the activity of Rho and Rac are coordinated around these receptors will be an important area for future study. It will also be crucial to examine the contribution of each GTPase pathway to exocytosis versus endocytosis of AMPARs, since both depend on the actin cytoskeleton.

Further clues into how signaling to the actin cytoskeleton regulates LTP/LTD can be gathered from recent studies of knockout animals which examine the role of actin signaling in regulating both synaptic plasticity and behavior. Several of these mice have targeted genes that correspond to human genes implicated in mental retardation (see also Review in this issue). One pathway that clearly regulates synaptic plasticity is signaling via Rac-PAK-LIMK-cofilin-actin, each of which is enriched in the postsynaptic spine and as discussed above regulates spine morphogenesis (Fig. 1c). Group I PAK (p21-kinase; composed of PAK1-3) is activated directly by binding to activated Rac or Cdc42 [130]. PAK3 null mice have mild behavioral deficits and impaired late phase LTP [131], yet spine morphology and density appeared normal in these animals. This is in contrast to mice expressing a dominant negative PAK (inhibits all PAK activity) transgene, which have enhanced LTP and impaired memory retention [34]. Differences between these mice may be due to functional compensation by PAK1, which is also expressed in the adult brain. Human mutations in PAK3, which are associated with mental retardation, may have dominant negative effects as well since they are truncations or missense mutations. Disruption of LIMK1, which is downstream of PAK and is mutated in Williams Syndrome, results in abnormal spine-head morphology and spatial learning deficits [40]. As discussed above, these effects are probably the result of reduced phosphorylation of the actin severing protein cofilin, which depending on the concentrations of cofilin, could result in elevated cofilin-mediated actin turnover.

A second pathway downstream of Rac is the RacWAVE1-Arp2/3-actin pathway (Fig. 1b). Again, each component of this signaling pathway is enriched in spines, and the net consequence of Arp2/3 activation via Rac and WAVE1 is an increase in branched actin filament polymerization [23, 132]. Disruption of WAVE1 leads to multiple behavioral abnormalities, including reduced anxiety, impaired psycho-motor function, and deficits in learning and memory [133]. Loss of WAVE1 also negatively impacts spinogenesis and results in altered bi-directional synaptic plasticity [31]. Compared to wildtype littermates, WAVE1 null mice display elevated LTP and reduced LTD. These alterations were associated with an increase in NMDA field responses relative to AMPA response, suggesting the composition of synaptic glutamate receptors is altered in these mice. Signals from Rac and Cdc42 can also be transduced to downstream targets within the spine via IRSp53 (Fig. 2i). IRSp53 contains an N-terminal I-BAR domain that recognizes outwardly protruding membranes and can bundle Factin, as well as a CRIB domain that binds Rac and Cdc42 [134]. At the C-terminal end IRSp53 contains an SH3 domain that can interact with several proteins including PSD95, Shank, WAVE2, N-WASP, and Eps8. Binding to these proteins also regulates the ability of IRSp53 to bundle Factin. Two recent studies of IRSp53 gene-trap mice show that loss of IRSp53 leads to elevated CA1 LTP and increased
NMDAR mediated synaptic transmission $[135,136]$. This increase in NMDAR synaptic transmission fits well with the observation that NR1 and NR2A/B subunit levels are increased in the PSD, whereas GluR1 levels were not. Null mice also display impairments in several behavioral tests, including spatial and non-spatial memory. Interestingly, knockout of the IRSp53 binding partner, Eps8 also increases NMDAR currents, but in cerebellar granule neurons [137]. Eps8 forms a complex with NR1, NR2A, and NR2C, and is known to associate with the Rac-GEF Sos-1 [137, 138]. Furthermore, Eps8 can directly regulate actin dynamics since it possesses barbed-end actin capping activity [139]. How these activities are coordinated to directly regulate NMDAR synaptic transmission is not clear. The behavioral effects of the loss of Eps8 are quite dramatic - null mice are resistant to the sedating effects of ethanol and consequently will voluntarily consume more compared to wildtype littermates [137]. NMDAR is a known target of ethanol and these data show that Eps8, by increasing NMDA currents, blunts the inhibitory effects of ethanol.

\section{CONCLUSION}

Over the last several years much progress has been made in understanding how actin dynamics influences the development (Fig. 1) and plasticity of excitatory synapses (Fig. 2). The combination of biochemistry with live imaging and genetically modified mice has made it possible to begin unifying evidence relating to spine-actin biology with complex behaviors. New advances in these areas will continue to push our knowledge of how actin-based signaling pathways influence these processes. It is clear that one important area will be to understand how different signaling pathways are organized within the spine to temporally and spatially coordinate synaptic physiology. Furthermore, it seems clear that actin signaling will also be important for synaptic development and plasticity beyond glutamatergic synapses. Understanding how actin regulates the development and function of other classes of synapses (for example inhibitory synapses) will be important for a clearer view of neural network integration.

\section{REFERENCES}

[1] Hering H, Sheng M. Dendritic spines: structure, dynamics and regulation. Nat Rev Neurosci 200; 2(12): 880-8.

[2] Calabrese B, Wilson MS, Halpain S. Development and regulation of dendritic spine synapses. Physiology (Bethesda). 2006; 21: 3847.

[3] Nimchinsky EA, Sabatini BL, Svoboda K. Structure and function of dendritic spines. Annu Rev Physiol 2002; 64: 313-53.

[4] Yuste R, Bonhoeffer T. Morphological changes in dendritic spines associated with long-term synaptic plasticity. Annu Rev Neurosci 2001; 24: 1071-89.

[5] Kasai H, Matsuzaki M, Noguchi J, Yasumatsu N, Nakahara H. Structure-stability-function relationships of dendritic spines. Trends Neurosci 2003; 26(7): 360-8.

[6] Newey SE, Velamoor V, Govek EE, Van Aelst L. Rho GTPases, dendritic structure, and mental retardation. J Neurobiol 2005; 64(1): 58-74.

[7] Ramakers GJ. Rho proteins, mental retardation and the cellular basis of cognition. Trends Neurosci 2002; 25(4): 191-9.

[8] Matus A. Actin-based plasticity in dendritic spines. Science 2000; 290(5492): 754-8.

[9] Symons MH, Mitchison TJ. Control of actin polymerization in live and permeabilized fibroblasts. J Cell Biol 1991; 114(3): 503-13.

[10] Wegner A. Head to tail polymerization of actin. J Mol Biol 1976;108(1):139-50.

[11] Pollard TD, Borisy GG. Cellular motility driven by assembly and disassembly of actin filaments. Cell 200; 112(4): 453-65. 
[12] Etienne-Manneville S, Hall A. Rho GTPases in cell biology. Nature 2002; 420(6916): 629-35.

[13] Hall A. Rho GTPases and the actin cytoskeleton. Science 1998; 279(5350): 509-14.

[14] Nakayama AY, Harms MB, Luo L. Small GTPases Rac and Rho in the maintenance of dendritic spines and branches in hippocampal pyramidal neurons. J Neurosci 2000; 20(14): 5329-38.

[15] Luo L, Hensch TK, Ackerman L, Barbel S, Jan LY, Jan YN. Differential effects of the Rac GTPase on Purkinje cell axons and dendritic trunks and spines. Nature 1996; 379(6568): 837-40.

[16] Tashiro A, Minden A, Yuste R. Regulation of dendritic spine morphology by the rho family of small GTPases: antagonistic roles of Rac and Rho. Cereb Cortex 2000; 10(10): 927-38.

[17] Tashiro A, Yuste R. Regulation of dendritic spine motility and stability by Rac1 and Rho kinase: evidence for two forms of spine motility. Mol Cell Neurosci 2004; 26(3): 429-40.

[18] Pilpel Y, Segal M. Activation of PKC induces rapid morphological plasticity in dendrites of hippocampal neurons via Rac and Rhodependent mechanisms. Eur J Neurosci 2004; 19(12): 3151-64.

[19] Soderling SH, Scott JD. WAVE signalling: from biochemistry to biology. Biochem Soc Trans 2006; 34(Pt 1): 73-6.

[20] Panchal SC, Kaiser DA, Torres E, Pollard TD, Rosen MK. A conserved amphipathic helix in WASP/Scar proteins is essential for activation of Arp2/3 complex. Nat Struct Biol 2003; 10(8): 591-8.

[21] Kim AS, Kakalis LT, Abdul-Manan N, Liu GA, Rosen MK. Autoinhibition and activation mechanisms of the Wiskott-Aldrich syndrome protein. Nature 2000; 404(6774): 151-8.

[22] Pollard TD. Regulation of actin filament assembly by Arp $2 / 3$ complex and formins. Annu Rev Biophys Biomol Struct 2007; 36: 45177.

[23] Soderling SH. Grab your partner with both hands: cytoskeletal remodeling by Arp2/3 signaling. Sci Signal 2009; 2(55): pe5.

[24] Racz B, Weinberg RJ. Organization of the Arp $2 / 3$ complex in hippocampal spines. J Neurosci 2008; 28(22): 5654-9.

[25] Irie F, Yamaguchi Y. EphB receptors regulate dendritic spine development via intersectin, $\mathrm{Cdc} 42$ and N-WASP. Nat Neurosci 2002; 5(11): 1117-8

[26] Wegner AM, Nebhan CA, Hu L, et al. N-wasp and the $\operatorname{arp} 2 / 3$ complex are critical regulators of actin in the development of dendritic spines and synapses. J Biol Chem 2008; 283(23): 15912-20.

[27] Eden S, Rohatgi R, Podtelejnikov AV, Mann M, Kirschner MW. Mechanism of regulation of WAVE1-induced actin nucleation by Rac1 and Nck. Nature 2002; 418(6899): 790-3.

[28] Ismail AM, Padrick SB, Chen B, Umetani J, Rosen MK. The WAVE regulatory complex is inhibited. Nat Struct Mol Biol 2009; 16(5): 561-3.

[29] Derivery E, Lombard B, Loew D, Gautreau A. The Wave complex is intrinsically inactive. Cell Motil Cytoskeleton 2009 [Epub ahead of print]

[30] Kim Y, Sung JY, Ceglia I, et al. Phosphorylation of WAVE1 regulates actin polymerization and dendritic spine morphology. Nature 2006; 442(7104): 814-7.

[31] Soderling SH, Guire ES, Kaech S, et al. A WAVE-1 and WRP signaling complex regulates spine density, synaptic plasticity, and memory. J Neurosci 2007; 27(2): 355-65.

[32] Edwards DC, Sanders LC, Bokoch GM, Gill GN. Activation of LIM-kinase by Pak1 couples Rac/Cdc42 GTPase signalling to actin cytoskeletal dynamics. Nat Cell Biol 1999; 1(5): 253-9.

[33] Bokoch GM. Biology of the p21-activated kinases. Annu Rev Biochem 2003; 72: 743-81.

[34] Hayashi ML, Choi SY, Rao BS, et al. Altered cortical synaptic morphology and impaired memory consolidation in forebrain- specific dominant-negative PAK transgenic mice. Neuron 2004; 42(5): 773-87.

[35] Schmandke A, Strittmatter SM. ROCK and Rho: biochemistry and neuronal functions of Rho-associated protein kinases. Neuroscientist 2007; 13(5): 454-69.

[36] Arber S, Barbayannis FA, Hanser H, et al. Regulation of actin dynamics through phosphorylation of cofilin by LIM-kinase. Nature 1998; 393(6687): 805-9.

[37] Yang N, Higuchi O, Ohashi K, et al. Cofilin phosphorylation by LIM-kinase 1 and its role in Rac-mediated actin reorganization. Nature 1998; 393(6687): 809-12.

[38] Maciver SK. How ADF/cofilin depolymerizes actin filaments? Curr Opin Cell Biol 1998; 10(1): 140-4.
[39] Racz B, Weinberg RJ. Spatial organization of cofilin in dendritic spines. Neuroscience 2006; 138(2): 447-56.

[40] Meng Y, Zhang Y, Tregoubov V, et al. Abnormal spine morphology and enhanced LTP in LIMK-1 knockout mice. Neuron 2002; 35(1): 121-33.

[41] Zhang W, Benson DL. Development and molecular organization of dendritic spines and their synapses. Hippocampus 2000; 10(5): 512-26.

[42] Carlisle HJ, Kennedy MB. Spine architecture and synaptic plasticity. Trends Neurosci 2005; 28(4): 182-7.

[43] Hotulainen P, Llano O, Smirnov S, et al. Defining mechanisms of actin polymerization and depolymerization during dendritic spine morphogenesis. J Cell Biol 2009; 185(2): 323-39.

[44] Chhabra ES, Higgs HN. The many faces of actin: matching assembly factors with cellular structures. Nat Cell Biol 2007; 9(10): $1110-21$

[45] Zuo Y, Lin A, Chang P, Gan WB. Development of long-term dendritic spine stability in diverse regions of cerebral cortex. Neuron 2005; 46(2): 181-9.

[46] Portera-Cailliau C, Pan DT, Yuste R. Activity-regulated dynamic behavior of early dendritic protrusions: evidence for different types of dendritic filopodia. J Neurosci 2003; 23(18): 7129-42.

[47] Maletic-Savatic M, Malinow R, Svoboda K. Rapid dendritic morphogenesis in CA1 hippocampal dendrites induced by synaptic activity. Science 1999; 283(5409): 1923-7.

[48] Soderling TR. CaM-kinases: modulators of synaptic plasticity. Curr Opin Neurobiol 2000; 10(3): 375-80.

[49] Jourdain P, Fukunaga K, Muller D. Calcium/calmodulin-dependent protein kinase II contributes to activity-dependent filopodia growth and spine formation. J Neurosci 2003; 23(33): 10645-9.

[50] Lohmann C, Bonhoeffer T. A role for local calcium signaling in rapid synaptic partner selection by dendritic filopodia. Neuron 2008; 59(2): 253-60

[51] Saneyoshi T, Wayman G, Fortin D, et al. Activity-dependent synaptogenesis: regulation by a CaM-kinase kinase/CaM-kinase I/betaPIX signaling complex. Neuron 2008; 57(1): 94-107.

[52] Klein R. Bidirectional modulation of synaptic functions by Eph/ephrin signaling. Nat Neurosci 2009; 12(1): 15-20.

[53] Henkemeyer M, Itkis OS, Ngo M, Hickmott PW, Ethell IM. Multiple EphB receptor tyrosine kinases shape dendritic spines in the hippocampus. J Cell Biol 2003; 163(6): 1313-26.

[54] Penzes P, Beeser A, Chernoff J, et al. Rapid induction of dendritic spine morphogenesis by trans-synaptic ephrinB-EphB receptor activation of the Rho-GEF kalirin. Neuron 2003; 37(2): 263-74.

[55] Tolias KF, Bikoff JB, Kane CG, Tolias CS, Hu L, Greenberg ME The Rac1 guanine nucleotide exchange factor Tiam1 mediates EphB receptor-dependent dendritic spine development. Proc Natl Acad Sci USA 2007; 104(17): 7265-70.

[56] Shi Y, Pontrello CG, DeFea KA, Reichardt LF, Ethell IM. Focal adhesion kinase acts downstream of EphB receptors to maintain mature dendritic spines by regulating cofilin activity. J Neurosci 2009; 29(25): 8129-42.

[57] Grunwald IC, Korte M, Adelmann G, et al. Hippocampal plasticity requires postsynaptic ephrinBs. Nat Neurosci 2004; 7(1): 33-40.

[58] Bouzioukh F, Wilkinson GA, Adelmann G, Frotscher M, Stein V, Klein R. Tyrosine phosphorylation sites in ephrinB2 are required for hippocampal long-term potentiation but not long-term depression. J Neurosci 2007; 27(42): 11279-88.

[59] Segura I, Essmann CL, Weinges S, Acker-Palmer A. Grb4 and GIT1 transduce ephrinB reverse signals modulating spine morphogenesis and synapse formation. Nat Neurosci 2007; 10(3): 301-10.

[60] Kayser MS, Nolt MJ, Dalva MB. EphB receptors couple dendritic filopodia motility to synapse formation. Neuron 2008; 59(1): 5669

[61] Takeichi M. The cadherin superfamily in neuronal connections and interactions. Nat Rev Neurosci 2007; 8(1): 11-20.

[62] Uchida N, Honjo Y, Johnson KR, Wheelock MJ, Takeichi M. The catenin/cadherin adhesion system is localized in synaptic junctions bordering transmitter release zones. J Cell Biol 1996; 135(3): 76779.

[63] Arikkath J. Regulation of dendrite and spine morphogenesis and plasticity by catenins. Mol Neurobiol 2009; 40(1): 46-54.

[64] Abe K, Chisaka O, Van Roy F, Takeichi M. Stability of dendritic spines and synaptic contacts is controlled by alpha N-catenin. Nat Neurosci 2004; 7(4): 357-63. 
[65] Okuda T, Yu LM, Cingolani LA, Kemler R, Goda Y. Beta-Catenin regulates excitatory postsynaptic strength at hippocampal synapses. Proc Natl Acad Sci USA 2007; 104(33): 13479-84.

[66] Abu-Elneel K, Ochiishi T, Medina M, et al. A delta-catenin signaling pathway leading to dendritic protrusions. J Biol Chem. 2008; 283(47): 32781-91.

[67] Arikkath J, Peng IF, Ng YG, et al. Delta-catenin regulates spine and synapse morphogenesis and function in hippocampal neurons during development. J Neurosci. 2009; 29(17): 5435-42.

[68] Naisbitt S, Kim E, Tu JC, et al. Shank, a novel family of postsynaptic density proteins that binds to the NMDA receptor/PSD95/GKAP complex and cortactin. Neuron 1999; 23(3): 569-82.

[69] Hering H, Sheng M. Activity-dependent redistribution and essential role of cortactin in dendritic spine morphogenesis. J Neurosci 2003; 23(37): 11759-69.

[70] Xie Z, Photowala H, Cahill ME, et al. Coordination of synaptic adhesion with dendritic spine remodeling by AF-6 and kalirin-7. J Neurosci 2008; 28(24): 6079-91.

[71] Yoshihara Y, De Roo M, Muller D. Dendritic spine formation and stabilization. Curr Opin Neurobiol 2009.

[72] Matsuno H, Okabe S, Mishina M, Yanagida T, Mori K, Yoshihara Y. Telencephalin slows spine maturation. J Neurosci 2006; 26(6): 1776-86.

[73] Furutani Y, Matsuno H, Kawasaki M, Sasaki T, Mori K, Yoshihara Y. Interaction between telencephalin and ERM family proteins mediates dendritic filopodia formation. J Neurosci 2007; 27(33): 8866-76.

[74] Bliss TV, Lomo T. Long-lasting potentiation of synaptic transmission in the dentate area of the anaesthetized rabbit following stimulation of the perforant path. J Physiol 1973; 232(2): 331-56.

[75] Fifkova E, Van Harreveld A. Long-lasting morphological changes in dendritic spines of dentate granular cells following stimulation of the entorhinal area. J Neurocytol 1977; 6(2): 211-30.

[76] Crick F. Do Dendritic Spines Twitch? Trends Neurosci 1982; 5: 44-6.

[77] Fifkova E, Delay RJ. Cytoplasmic actin in neuronal processes as a possible mediator of synaptic plasticity. J Cell Biol 1982; 95(1): 345-50.

[78] Matus A, Ackermann M, Pehling G, Byers HR, Fujiwara K. High actin concentrations in brain dendritic spines and postsynaptic densities. Proc Natl Acad Sci USA 1982; 79(23): 7590-4.

[79] Dudek SM, Bear MF. Homosynaptic long-term depression in area CA1 of hippocampus and effects of N-methyl-D-aspartate receptor blockade. Proc Natl Acad Sci USA 1992; 89(10): 4363-7.

[80] Fukazawa Y, Saitoh Y, Ozawa F, Ohta Y, Mizuno K, Inokuchi K. Hippocampal LTP is accompanied by enhanced F-actin content within the dendritic spine that is essential for late LTP maintenance in vivo. Neuron 2003; 38(3): 447-60.

[81] Krucker T, Siggins GR, Halpain S. Dynamic actin filaments are required for stable long-term potentiation (LTP) in area CA1 of the hippocampus. Proc Natl Acad Sci USA 2000; 97(12): 6856-61.

[82] Kim CH, Lisman JE. A role of actin filament in synaptic transmission and long-term potentiation. J Neurosci 1999; 19(11): 4314-24.

[83] Matsuzaki M, Honkura N, Ellis-Davies GC, Kasai H. Structural basis of long-term potentiation in single dendritic spines. Nature 2004; 429(6993): 761-6.

[84] Rostaing P, Real E, Siksou L, et al. Analysis of synaptic ultrastructure without fixative using high-pressure freezing and tomography. Eur J Neurosci 2006; 24(12): 3463-74.

[85] Star EN, Kwiatkowski DJ, Murthy VN. Rapid turnover of actin in dendritic spines and its regulation by activity. Nat Neurosci 2002; 5(3): 239-46.

[86] Lin B, Kramar EA, Bi X, Brucher FA, Gall CM, Lynch G. Theta stimulation polymerizes actin in dendritic spines of hippocampus. $\mathrm{J}$ Neurosci 2005; 25(8): 2062-9.

[87] Kramar EA, Lin B, Rex CS, Gall CM, Lynch G. Integrin-driven actin polymerization consolidates long-term potentiation. Proc Natl Acad Sci USA 2006; 103(14): 5579-84.

[88] Okamoto K, Nagai T, Miyawaki A, Hayashi Y. Rapid and persistent modulation of actin dynamics regulates postsynaptic reorganization underlying bidirectional plasticity. Nat Neurosci 2004; 7(10): 1104-12.

[89] Honkura N, Matsuzaki M, Noguchi J, Ellis-Davies GC, Kasai H. The subspine organization of actin fibers regulates the structure and plasticity of dendritic spines. Neuron 2008; 57(5): 719-29.
[90] Rall W. Studies in Neurophysiology. Porter R, Ed. Cambridge: Cambridge University Press 1978.

[91] Racz B, Weinberg RJ. The subcellular organization of cortactin in hippocampus. J Neurosci 2004; 24(46): 10310-7.

[92] Neuhoff H, Sassoe-Pognetto M, Panzanelli P, Maas C, Witke W, Kneussel M. The actin-binding protein profilin I is localized at synaptic sites in an activity-regulated manner. Eur J Neurosci 2005; 21(1): 15-25.

[93] Ackermann M, Matus A. Activity-induced targeting of profilin and stabilization of dendritic spine morphology. Nat Neurosci 2003; 6(11): 1194-200.

[94] Lamprecht R, Farb CR, Rodrigues SM, LeDoux JE. Fear conditioning drives profilin into amygdala dendritic spines. Nat Neurosci 2006; 9(4): 481-3.

[95] Billuart P, Bienvenu T, Ronce N, et al. Oligophrenin 1 encodes a rho-GAP protein involved in X-linked mental retardation. Pathol Biol (Paris) 199; 46(9): 678.

[96] Nadif KN, Nakano-Kobayashi A, Malinow R, Li B, Van Aelst L. The Rho-linked mental retardation protein oligophrenin-1 controls synapse maturation and plasticity by stabilizing AMPA receptors. Genes Dev 2009; 23(11): 1289-302.

[97] Kessels HW, Malinow R. Synaptic AMPA receptor plasticity and behavior. Neuron 2009; 61(3): 340-50.

[98] Derkach VA, Oh MC, Guire ES, Soderling TR. Regulatory mechanisms of AMPA receptors in synaptic plasticity. Nat Rev Neurosci 2007; 8(2): 101-13.

[99] Newpher TM, Ehlers MD. Glutamate receptor dynamics in dendritic microdomains. Neuron 2008; 58(4): 472-97.

[100] Malinow R, Malenka RC. AMPA receptor trafficking and synaptic plasticity. Annu Rev Neurosci 2002; 25: 103-26.

[101] Ashby MC, De La Rue SA, Ralph GS, Uney J, Collingridge GL, Henley JM. Removal of AMPA receptors (AMPARs) from synapses is preceded by transient endocytosis of extrasynaptic AMPARs. J Neurosci 2004; 24(22): 5172-6.

[102] Snyder EM, Philpot BD, Huber KM, Dong X, Fallon JR, Bear MF. Internalization of ionotropic glutamate receptors in response to mGluR activation. Nat Neurosci 2001; 4(11): 1079-85.

[103] Heynen AJ, Quinlan EM, Bae DC, Bear MF. Bidirectional, activity-dependent regulation of glutamate receptors in the adult hippocampus in vivo. Neuron 2000; 28(2): 527-36.

[104] Montgomery JM, Madison DV. State-dependent heterogeneity in synaptic depression between pyramidal cell pairs. Neuron 2002; 33(5): 765-77.

[105] Collingridge GL, Isaac JT, Wang YT. Receptor trafficking and synaptic plasticity. Nat Rev Neurosci 2004; 5(12): 952-62.

[106] Carroll RC, Beattie EC, von Zastrow M, Malenka RC. Role of AMPA receptor endocytosis in synaptic plasticity. Nat Rev Neurosci 2001; 2(5): 315-24.

[107] Chowdhury S, Shepherd JD, Okuno H, et al. Arc/Arg3.1 interacts with the endocytic machinery to regulate AMPA receptor trafficking. Neuron 2006; 52(3): 445-59.

[108] Wyszynski M, Lin J, Rao A, et al. Competitive binding of alphaactinin and calmodulin to the NMDA receptor. Nature 1997; 385(6615): 439-42.

[109] Wyszynski M, Kharazia V, Shanghvi R, et al. Differential regional expression and ultrastructural localization of alpha-actinin-2, a putative NMDA receptor-anchoring protein, in rat brain. J Neurosci 1998; 18(4): 1383-92.

[110] Scott DB, Michailidis I, Mu Y, Logothetis D, Ehlers MD. Endocytosis and degradative sorting of NMDA receptors by conserved membrane-proximal signals. J Neurosci 2004; 24(32): 7096-109.

[111] Rosenmund C, Westbrook GL. Calcium-induced actin depolymerization reduces NMDA channel activity. Neuron 1993; 10(5): 80514.

[112] Norenberg W, Hofmann F, Illes P, Aktories K, Meyer DK. Rundown of somatodendritic N-methyl-D-aspartate (NMDA) receptor channels in rat hippocampal neurones: evidence for a role of the small GTPase RhoA. Br J Pharmacol 1999; 127(5): 1060-3.

[113] Nakazawa T, Watabe AM, Tezuka T, et al. p250GAP, a novel brain-enriched GTPase-activating protein for Rho family GTPases, is involved in the N-methyl-d-aspartate receptor signaling. Mol Biol Cell 2003; 14(7): 2921-34.

[114] Nakazawa T, Kuriu T, Tezuka T, Umemori H, Okabe S, Yamamoto T. Regulation of dendritic spine morphology by an NMDA receptor-associated Rho GTPase-activating protein, p250GAP. J Neurochem 2008; 105(4): 1384-93. 
[115] Wayman GA, Davare M, Ando H, et al. An activity-regulated microRNA controls dendritic plasticity by down-regulating p250GAP. Proc Natl Acad Sci USA 2008; 105(26): 9093-8.

[116] Shen L, Liang F, Walensky LD, Huganir RL. Regulation of AMPA receptor GluR1 subunit surface expression by a 4 . 1N-linked actin cytoskeletal association. J Neurosci 2000; 20(21): 7932-40.

[117] Zhou Q, Xiao M, Nicoll RA. Contribution of cytoskeleton to the internalization of AMPA receptors. Proc Natl Acad Sci USA 2001; 98(3): 1261-6.

[118] Lin DT, Makino Y, Sharma K, et al. Regulation of AMPA receptor extrasynaptic insertion by $4.1 \mathrm{~N}$, phosphorylation and palmitoylation. Nat Neurosci 2009; 12(7): 879-87.

[119] Park M, Penick EC, Edwards JG, Kauer JA, Ehlers MD. Recycling endosomes supply AMPA receptors for LTP. Science 2004; 305(5692): 1972-5.

[120] Wang Z, Edwards JG, Riley N, et al. Myosin Vb mobilizes recycling endosomes and AMPA receptors for postsynaptic plasticity. Cell 2008; 135(3): 535-48.

[121] Kim CH, Chung HJ, Lee HK, Huganir RL. Interaction of the AMPA receptor subunit GluR2/3 with PDZ domains regulates hippocampal long-term depression. Proc Natl Acad Sci USA 2001; 98(20): 11725-30.

[122] Iwakura Y, Nagano T, Kawamura M, et al. N-methyl-D-aspartateinduced alpha-amino-3-hydroxy-5-methyl-4-isoxazoleproprionic acid (AMPA) receptor down-regulation involves interaction of the carboxyl terminus of GluR2/3 with Pick1. Ligand-binding studies using Sindbis vectors carrying AMPA receptor decoys. J Biol Chem 2001; 276(43): 40025-32.

[123] Hanley JG, Henley JM. PICK1 is a calcium-sensor for NMDAinduced AMPA receptor trafficking. EMBO J 2005; 24(18): 326678.

[124] Rocca DL, Martin S, Jenkins EL, Hanley JG. Inhibition of Arp2/3mediated actin polymerization by PICK 1 regulates neuronal morphology and AMPA receptor endocytosis. Nat Cell Biol 2008; 10: 259-71.

[125] Merrifield CJ, Feldman ME, Wan L, Almers W. Imaging actin and dynamin recruitment during invagination of single clathrin-coated pits. Nat Cell Biol 2002; 4(9): 691-8.

[126] Merrifield CJ, Qualmann B, Kessels MM, Almers W. Neural Wiskott Aldrich Syndrome Protein (N-WASP) and the Arp2/3 complex are recruited to sites of clathrin-mediated endocytosis in cultured fibroblasts. Eur J Cell Biol 2004; 83(1): 13-8.
[127] Benesch S, Polo S, Lai FP, et al. N-WASP deficiency impairs EGF internalization and actin assembly at clathrin-coated pits. J Cell Sci 2005; 118(Pt 14): 3103-15.

[128] Billuart P, Bienvenu T, Ronce N, et al. Oligophrenin-1 encodes a rhoGAP protein involved in $\mathrm{X}$-linked mental retardation. Nature 1998; 392(6679): 923-6.

[129] Xie Z, Srivastava DP, Photowala H, et al. Kalirin-7 controls activity-dependent structural and functional plasticity of dendritic spines. Neuron 2007; 56(4): 640-56.

[130] Kreis P, Barnier JV. PAK signalling in neuronal physiology. Cell Signal 2009; 21(3): 384-93.

[131] Meng J, Meng Y, Hanna A, Janus C, Jia Z. Abnormal long-lasting synaptic plasticity and cognition in mice lacking the mental retardation gene Pak3. J Neurosci 2005; 25(28): 6641-50.

[132] Welch MD. The world according to Arp: regulation of actin nucleation by the Arp2/3 complex. Trends Cell Biol 1999; 9(11): 4237.

[133] Soderling SH, Langeberg LK, Soderling JA, et al. Loss of WAVE1 causes sensorimotor retardation and reduced learning and memory in mice. Proc Natl Acad Sci USA 2003;100(4):1723-8.

[134] Scita G, Confalonieri S, Lappalainen P, Suetsugu S. IRSp53: crossing the road of membrane and actin dynamics in the formation of membrane protrusions. Trends Cell Biol 2008; 18(2): 52-60.

[135] Kim MH, Choi J, Yang J, et al. Enhanced NMDA receptormediated synaptic transmission, enhanced long-term potentiation, and impaired learning and memory in mice lacking IRSp53. J Neurosci 2009; 29(5): 1586-95.

[136] Sawallisch C, Berhorster K, Disanza A, et al. The insulin receptor substrate of $53 \mathrm{kDa}$ (IRSp53) limits hippocampal synaptic plasticity. J Biol Chem 2009; 284(14): 9225-36.

[137] Offenhauser N, Castelletti D, Mapelli L, et al. Increased ethanol resistance and consumption in Eps8 knockout mice correlates with altered actin dynamics. Cell 2006; 127(1): 213-26.

[138] Innocenti M, Frittoli E, Ponzanelli I, et al. Phosphoinositide 3kinase activates Rac by entering in a complex with Eps8, Abi1, and Sos-1. J Cell Biol 2003; 160(1): 17-23.

[139] Disanza A, Carlier MF, Stradal TE, et al. Eps8 controls actin-based motility by capping the barbed ends of actin filaments. Nat Cell Biol 2004; 6(12): 1180-8.

(C) Okada and Soderling; Licensee Bentham Open.

This is an open access article licensed under the terms of the Creative Commons Attribution Non-Commercial License (http://creativecommons.org/licenses/by-nc/3.0/) which permits unrestricted, non-commercial use, distribution and reproduction in any medium, provided the work is properly cited. 